Article

\title{
Topological Properties of $E$-Metric Spaces with Applications to Fixed Point Theory
}

\author{
Huaping Huang \\ School of Mathematics and Statistics, Chongqing Three Gorges University, Wanzhou 404020, China; \\ mathhhp@163.com
}

Received: 24 October 2019; Accepted: 6 December 2019; Published: 11 December 2019

check for updates

\begin{abstract}
The purpose of this paper is to present some topological properties in $E$-metric spaces such as the properties of $e$-sequences, the decision conditions of $e$-Cauchy sequences, the characteristics of non-normal cones, and so on. Moreover, the theorem of nested closed-balls in such spaces is displayed. In addition, some principal applications to fixed point theory are also given.
\end{abstract}

Keywords: $E$-metric space; semi-interior point; $e$-sequence; $e$-Cauchy sequence; fixed point; $T$-stable

\section{Introduction}

Over the past several decades, nonlinear functional analysis, especially fixed point theory in ordered normed spaces had covered a large number of applications in optimization theory, game theory, variational inequalities, dynamical systems, fractals, graph theory, models in economy, computer science, and many other fields. Among them a partial ordering, is given by utilizing vector cones from certain spaces. Using this partial ordering, certain elements may be compared better than crude estimates via a norm. By substituting an ordered Banach space instead of the real line, in 2007, Huang and Zhang [1] introduced the concept of cone metric space with a new point of view. They considered convergent and Cauchy sequences in terms of interior points with regard to the underlying cone partial ordering. Subsequently, many mathematicians paid their attention to fixed point problems in such spaces (see [2-7] and the references therein).

In 2012, Rawashdeh et al. [8] modified the definition of cone metric space by means of using an ordered vector space instead of the ordered Banach space, and introduced the notion of $E$-metric space. They also dealt with convergent and Cauchy sequences via interior points regarding the same cone partial ordering as the mentioned above. It is worth pointing out that most fixed point problems from cone metric spaces and $E$-metric spaces are embedded into solid cones, whereas, solid cones are so-called cones containing at least one interior point. Fixed point results in the framework of these spaces frequently rely on the solid cones. As a matter of fact, non-solid cones exist a great deal (see [2,5]). As a consequence, these results inevitably lead to limitations in applications.

Fortunately, in 2017, Basile et al. [9] introduced the notion of the semi-interior point, and took into account fixed point results in $E$-metric spaces by embedding non-solid cones in which the cones contain semi-interior points. In 2019, based on [8,9], Mehmood et al. [10] obtained some fixed results in the setting of $E$-metric spaces by embedding such cones. The topological properties in cone metric spaces are becoming the center of strong research activities in recent years (see [11-14]). Using the topological properties of certain spaces, we are able to have an insight into the interior constructions of spaces. Therefore, it is valuable for us to investigate the topological properties.

To the best of our knowledge, we are the first to focus on systemic investigations on topological properties in $E$-metric spaces. Throughout this paper, we give some basic properties in $E$-metric spaces with regard to cones containing semi-interior points. By using the properties obtained, we learn the substantive characteristics of $E$-metric spaces. Furthermore, as applications, we cope with a class of 
fixed point problems such as the existence and uniqueness of fixed points for Hardy-Rogers type mapping, the T-stability of Picard's iteration and the equivalence between two distinct $e$-sequences in such spaces. All the results obtained in this paper will play a significant role in forthcoming research.

The following definitions and results will be needed in this paper.

Definition 1 ([1]). Let $E$ be a real normed space, $E^{+}$be a nonempty closed and convex subset of $E$, and $\theta_{E}$ be a zero element in $E$. Then $E^{+}$is called a positive cone if it satisfies

(1) $x \in E^{+}$and $\alpha \geq 0$ imply $\alpha x \in E^{+}$;

(2) $x \in E^{+}$and $-x \in E^{+}$imply $x=\theta_{E}$.

Definition 2 ([1]). Let $E$ be a real normed space and $E^{+}$a positive cone in $E$. We say $\preceq$ is a partial ordering relation on $E$ if

$$
x, y \in E, x \preceq y \text { if and only if } y-x \in E^{+} .
$$

Clearly,

$$
x \in E^{+} \text {if and only if } \theta_{E} \preceq x .
$$

Definition 3 ([8]). A real normed space $E$ with a norm $\|\cdot\|$ is called a real ordered vector space if the following conditions hold:

(i) $x, y, z \in E$ and $x \preceq y$ imply $x+z \preceq y+z$;

(ii) $\alpha \geq 0, x \in E$ and $\theta_{E} \preceq x$ imply $\theta_{E} \preceq \alpha x$.

In the sequel, unless there is a special explanation, we always denote by $\mathbb{N}$, the set of positive integers. We also denote by $E$, the real ordered vector space, $E^{+}$, the positive cone in $E$, and int $E^{+}$, the interior of $E^{+}$. We say that

$$
U=\{x \in E:\|x\| \leq 1\}
$$

is the closed unit ball of $E$ and that

$$
U_{+}=U \cap E^{+}
$$

is the positive part of $U$.

Definition 4 ([1]). $E^{+}$is called:

(1) a solid cone if int $E^{+} \neq \varnothing$;

(2) a normal cone if there exists an $M>0$ such that

$$
x, y \in E \text { and } \theta_{E} \preceq x \preceq y \text { imply }\|x\| \leq M\|x\| .
$$

The least positive number satisfying the above is called the normal constant of $E^{+}$.

Definition 5 ([8]). Let $X$ be a nonempty set and $d^{E}: X \times X \rightarrow E$ a mapping. Then $d^{E}$ is called an E-metric on $X$ if for all $x, y, z \in X$, it satisfies

$$
\begin{aligned}
& \text { (1) } \theta_{E} \preceq d^{E}(x, y), d^{E}(x, y)=\theta_{E} \text { if and only if } x=y \text {; } \\
& \text { (2) } d^{E}(x, y)=d^{E}(y, x) ; \\
& \text { (3) } d^{E}(x, y) \preceq d^{E}(x, z)+d^{E}(z, y) .
\end{aligned}
$$

The pair $\left(X, d^{E}\right)$ is called an E-metric space.

Definition 6 ([9]). The point $x_{0} \in E^{+}$is called a semi-interior point of $E^{+}$if there exits a real number $\rho>0$ such that

$$
x_{0}-\rho U_{+} \subseteq E^{+} .
$$


Here and thereafter, denote by $\left(E^{+}\right)^{\ominus}$ the set of all semi-interior points of $E^{+}$. We say that $\lll$ is a partial relation on $E^{+}$if

$$
x, y \in E^{+} \text {and } x \lll y \text { if and only if } y-x \in\left(E^{+}\right)^{\ominus} \text {. }
$$

It is easy to see that

$$
x \in\left(E^{+}\right)^{\ominus} \text { if and only if } \theta_{E} \lll x .
$$

Any interior point of $E^{+}$is a semi-interior point. However, the converse is not true. See Example 2.5 of [9].

Definition 7 ([10]). Let $\left(E^{+}\right)^{\ominus} \neq \varnothing$ and $\left(X, d^{E}\right)$ be an E-metric space. Let $\left\{x_{n}\right\}$ be a sequence in $X$ and $x \in X$. We say

(i) $\left\{x_{n}\right\}$ is e-convergent to $x$ if for any $e \ggg \theta_{E}$, there exists a natural number $N$ such that $d^{E}\left(x_{n}, x\right) \lll e$ for all $n>N$;

(ii) $\left\{x_{n}\right\}$ is an e-Cauchy sequence if for any $e \ggg \theta_{E}$, there exists a natural number $N$ such that $d^{E}\left(x_{n}, x_{m}\right) \lll$ e for all $n, m>N$;

(iii) $\left(X, d^{E}\right)$ is e-complete if every e-Cauchy sequence is e-convergent in $X$.

Lemma 1 ([5]). Let $0 \leq \lambda<1$ be a constant, $u \in E^{+}$and $u \preceq \lambda u$. Then $u=\theta_{E}$.

\section{Main Results}

In this section, omitting the assumption of solid cones of the main results in the existing literature, we shall give some topological properties relevant to semi-interior points in $E$-metric spaces. It will help us apperceive the internal structure of the spaces.

Proposition 1. Let $x, y \in E$. Then $x \lll y$ implies $x \preceq y$.

Proof. Let $x, y \in E$ and $x \lll y$. In view of $x \lll y$, then $y-x \in\left(E^{+}\right)^{\ominus}$, so there exists $\rho>0$ such that

$$
y-x-\rho U_{+} \subseteq E^{+}
$$

Noting that $\theta_{E} \in U_{+}$, it follows that

$$
y-x=y-x-\theta_{E}=y-x-\rho \theta_{E} \in y-x-\rho U_{+} \subseteq E^{+},
$$

which means that $x \preceq y$.

Proposition 2. Let $x \in \operatorname{int} E^{+}$and $y \in E$ such that $x \lll y$. Then $y \in$ int $E^{+}$.

Proof. On account of $x \lll y$, by Proposition 1 , we have $x \preceq y$, further, $y-x \in E^{+}$. As $x \in \operatorname{int} E^{+} \triangleq U$ implies $x \in E^{+}$, then $y=x+(y-x) \in E^{+}$, here and thereafter " $\triangleq "$ means "denote and equal". Let

$$
f: E \mapsto E \quad(t \mapsto t+x-y)
$$

be a mapping. In view of $f(y)=x \in U$, it means that $U$ is the image set of $f$. Noting that $f$ is continuous and $U$ is an open set, it follows that $f^{-1}(U)$ is also an open set. Thereby we only need to prove $f^{-1}(U) \subseteq E^{+}$. This is because if $f^{-1}(U) \subseteq E^{+}$, then $y \in U$, based on the fact that $y \in f^{-1}(U)$.

Indeed, since $f$ is a bijection, then $f^{-1}(U)=\left\{f^{-1}(u): u \in U\right\}$ is well-defined. Hence, $f^{-1}(u)=u+(y-x) \in E^{+}$if $u \in U \subseteq E^{+}$. Therefore, $f^{-1}(U) \subseteq E^{+}$. 
Proposition 3. Let $x, y, z \in E$. Then $x \lll z$ if one of the following holds:

$$
x \preceq y \lll z, \quad x \lll y \preceq z, \quad x \lll y \lll z .
$$

Proof. Let $x, y, z \in E$. Without loss of generality, assume that $x \preceq y \lll z$. Since $x \preceq y$, then $y-x \in E^{+}$. By virtue of $y \lll z$, i.e., $z-y \in\left(E^{+}\right)^{\ominus}$, hence there exists $\rho>0$ such that

$$
z-y-\rho U_{+} \subseteq E^{+} .
$$

Noting that $E^{+}$is a positive cone, it follows that

$$
z-x-\rho U_{+}=\left(z-y-\rho U_{+}\right)+(y-x) \subseteq E^{+},
$$

thus, $z-x \in\left(E^{+}\right)^{\ominus}$, that is, $x \lll z$. Similarly, we may prove the remaining cases.

Proposition 4. $E^{+}+\left(E^{+}\right)^{\ominus}=\left(E^{+}\right)^{\ominus}$.

Proof. Choose $x \in E^{+}, y \in\left(E^{+}\right)^{\ominus}$. On the one hand, it is valid that $\theta_{E} \preceq x, \theta_{E} \lll y$. Hence,

$$
\theta_{E} \lll y=\theta_{E}+y \preceq x+y .
$$

Thus by Proposition 3, it follows that $\theta_{E} \lll x+y$, i.e., $x+y \in\left(E^{+}\right)^{\ominus}$. That is to say,

$$
E^{+}+\left(E^{+}\right)^{\ominus} \subseteq\left(E^{+}\right)^{\ominus} .
$$

On the other hand, due to $\theta_{E} \in E^{+}$and $y \in\left(E^{+}\right)^{\ominus}$, then

$$
y=\theta_{E}+y \in E^{+}+\left(E^{+}\right)^{\ominus} .
$$

Consequently,

$$
\left(E^{+}\right)^{\ominus} \subseteq E^{+}+\left(E^{+}\right)^{\ominus}
$$

Considering (1) and (2), we demonstrate that

$$
E^{+}+\left(E^{+}\right)^{\ominus}=\left(E^{+}\right)^{\ominus}
$$

The proof is completed.

Proposition 5. Let $\lambda>0$ be a real number. Then $\lambda\left(E^{+}\right)^{\ominus}=\left(E^{+}\right)^{\ominus}$.

Proof. Choose $x \in\left(E^{+}\right)^{\ominus}$. Then there exists a real number $\rho>0$ such that

$$
x-\rho U_{+} \subseteq E^{+} .
$$

Since $\lambda>0$ and $E^{+}$is a positive cone, then

$$
\lambda x-\lambda \rho U_{+}=\lambda\left(x-\rho U_{+}\right) \subseteq E^{+} .
$$

Taking $\rho_{1}=\lambda \rho$, (3) becomes

$$
\lambda x-\rho_{1} U_{+} \subseteq E^{+} .
$$


Hence, $\lambda x$ is a semi-interior point in $E^{+}$, that is, $\lambda x \in\left(E^{+}\right)^{\ominus}$. This leads to

$$
\lambda\left(E^{+}\right)^{\ominus} \subseteq\left(E^{+}\right)^{\ominus} .
$$

Now we show that

$$
\left(E^{+}\right)^{\ominus} \subseteq \lambda\left(E^{+}\right)^{\ominus}
$$

Indeed, by (4), we get

$$
\left(E^{+}\right)^{\ominus}=\lambda \cdot \frac{1}{\lambda}\left(E^{+}\right)^{\ominus} \subseteq \lambda\left(E^{+}\right)^{\ominus} .
$$

Combining (4) and (5), the claims holds.

Remark 1. Clearly,

$$
\left(E^{+}\right)^{\ominus}+\left(E^{+}\right)^{\ominus}=\left(E^{+}\right)^{\ominus} .
$$

Indeed, by Proposition 5, it follows that

$$
\left(E^{+}\right)^{\ominus}+\left(E^{+}\right)^{\ominus}=2\left(E^{+}\right)^{\ominus}=\left(E^{+}\right)^{\ominus} .
$$

Proposition 6. Let $0<\alpha \leq \beta$ and $\theta_{E} \preceq x \lll y$. Then $\alpha x \lll \beta y$. In particular, $\alpha x \lll \alpha y$.

Proof. By $\theta_{E} \preceq x \lll y$, it follows that $x \in E^{+}$and $y-x \in\left(E^{+}\right)^{\ominus}$. Since $0<\alpha \leq \beta$, then by Proposition 5 and Definition 1, it may be verified that

$$
\beta(y-x) \in\left(E^{+}\right)^{\ominus}, \quad(\beta-\alpha) x \in E^{+} .
$$

Thus, by (6) and Proposition 4, we obtain

$$
\beta y-\alpha x=\beta(y-x)+(\beta-\alpha) x \in\left(E^{+}\right)^{\ominus},
$$

which implies that $\alpha x \lll \beta y$.

Proposition 7. If $\theta_{E} \preceq u \lll e$ holds for any $e \in\left(E^{+}\right)^{\ominus}$, then $u=\theta_{E}$.

Proof. Due to $e \in\left(E^{+}\right)^{\ominus}$, then by Proposition 5, it follows that $\frac{e}{n} \in\left(E^{+}\right)^{\ominus}$ for any $n \in \mathbb{N}$. Thus, by the hypothesis, we have $u \lll \frac{e}{n}$, which implies that $\frac{e}{n}-u \in\left(E^{+}\right)^{\ominus} \subseteq E^{+}$. Let $n$ tend to $\infty$ and notice that $E^{+}$is a closed set. Then $-u \in E^{+}$. Now that $\theta_{E} \preceq u$ leads to $u \in E^{+}$, hence, $u=\theta_{E}$.

Proposition 8. $\left(E^{+}\right)^{\ominus}$ is a closed set.

Proof. Let $\left\{x_{n}\right\}$ be a sequence of $\left(E^{+}\right)^{\ominus}$ such that $x_{n} \rightarrow x \in E$ as $n \rightarrow \infty$. In view of $x_{n} \in\left(E^{+}\right)^{\ominus}$, then there exists a real number $\rho>0$ such that $x_{n}-\rho U_{+} \subseteq E^{+}$. Taking the limit as $n \rightarrow \infty$ from this item together with the fact that $E^{+}$is a closed set, it follows that $x-\rho U_{+} \subseteq E^{+}$. This means that $x \in\left(E^{+}\right)^{\ominus}$. In other words, $\left(E^{+}\right)^{\ominus}$ is a closed set.

Proposition 9. If $a \lll b+e$ holds for any $e \in\left(E^{+}\right)^{\ominus}$, then $a \lll b$.

Proof. Since $a \lll b+e$ holds for any $e \in\left(E^{+}\right)^{\ominus}$, and $e \in\left(E^{+}\right)^{\ominus}$, it follows from Proposition 5 that $\frac{e}{n} \in\left(E^{+}\right)^{\ominus}$ is satisfied for any $n \in \mathbb{N}$, which means that $a \lll b+\frac{e}{n}$ holds for any $n \in \mathbb{N}$. Thus, 
$b-a+\frac{e}{n} \in\left(E^{+}\right)^{\ominus}$ for any $n \in \mathbb{N}$. Passing to the limit as $n \rightarrow \infty$ from this item together with Proposition 8 , we have $b-a \in\left(E^{+}\right)^{\ominus}$, i.e., $a \lll b$.

Proposition 10. Let $x \in E$ and $y \in\left(E^{+}\right)^{\ominus}$. Then there exists $m \in \mathbb{N}$ such that $x \lll m y$.

Proof. Owing to

$$
\lim _{n \rightarrow \infty}\left(y-\frac{x}{n}\right)=y \in\left(E^{+}\right)^{\ominus}
$$

then by Proposition 8 , there exists $m \in \mathbb{N}$ such that $y-\frac{x}{m} \in\left(E^{+}\right)^{\ominus}$. Thus, it follows immediately from Proposition 5 that

$$
m y-x=m\left(y-\frac{x}{m}\right) \in\left(E^{+}\right)^{\ominus} .
$$

Therefore, $x \lll m y$ holds.

Motivated by the concept of $c$-sequence from [15], we introduce the concept of the $e$-sequence in E-metric space as follows.

Definition 8. A sequence $\left\{x_{n}\right\}$ in $E^{+}$is called an e-sequence if for eache $\ggg \theta_{E}$, there exists $N \in \mathbb{N}$ such that $x_{n} \lll e$ for all $n>N$.

Proposition 11. Let $\left\{x_{n}\right\}$ and $\left\{y_{n}\right\}$ be e-sequences in $E$ and $\alpha, \beta \geq 0$ be constants. Then $\left\{\alpha x_{n}+\beta y_{n}\right\}$ is an e-sequence.

Proof. Without loss of generality, we assume that $\alpha, \beta>0$. Since $\left\{x_{n}\right\}$ is an $e$-sequence, then for any $e \ggg \theta_{E}$, there exists $N_{1} \in \mathbb{N}$ such that $x_{n} \lll \frac{1}{2 \alpha} e$ for all $n>N_{1}$. Similarly, since $\left\{y_{n}\right\}$ is an $e$-sequence, then for the previously mentioned $e \ggg \theta_{E}$, there exists $N_{2} \in \mathbb{N}$ such that $y_{n} \lll \frac{1}{2 \beta} e$ for all $n>N_{2}$. We make $N=\max \left\{N_{1}, N_{2}\right\}$, then for all $n>N$, by Proposition 6, we have

$$
\alpha x_{n}+\beta y_{n} \lll \alpha \cdot \frac{1}{2 \alpha} e+\beta \cdot \frac{1}{2 \beta} e=e,
$$

therefore, $\left\{\alpha x_{n}+\beta y_{n}\right\}$ is an $e$-sequence.

Proposition 12. Let $\left\{x_{n}\right\}$ be a sequence in $E$ and $x_{n} \rightarrow \theta_{E}(n \rightarrow \infty)$. Then $\left\{x_{n}\right\}$ is an e-sequence.

Proof. For any $e \in\left(E^{+}\right)^{\ominus}$, there exists a real number $\rho_{1}>0$ such that

$$
e-\rho_{1} U_{+} \subseteq E^{+}
$$

Since $x_{n} \rightarrow \theta_{E}(n \rightarrow \infty)$, then there exist $N \in \mathbb{N}$ and a real number $\rho_{2}>\rho_{1}$ such that $x_{n} \in \frac{\rho_{2}}{2} U_{+}$for all $n>N$. Hence, we have

$$
e-x_{n}-\frac{\rho_{2}}{2} U_{+} \subseteq e-\frac{\rho_{2}}{2} U_{+}-\frac{\rho_{2}}{2} U_{+}=e-\rho_{2} U_{+} \subseteq e-\rho_{1} U_{+} \subseteq E^{+},
$$

from which, it follows that

$$
e-x_{n} \in\left(E^{+}\right)^{\ominus}(n>N)
$$

so $x_{n} \lll e(n>N)$.

Proposition 13. Let $\left\{x_{n}\right\}$ and $\left\{y_{n}\right\}$ be two sequences in $E$ such that $x_{n} \preceq y_{n}$ and $y_{n} \rightarrow \theta_{E}(n \rightarrow \infty)$. Then $\left\{x_{n}\right\}$ is an e-sequence. 
Proof. Since $y_{n} \rightarrow \theta_{E}(n \rightarrow \infty)$, then by Proposition 12 , for any $e \in\left(E^{+}\right)^{\ominus}$, there exists $N \in \mathbb{N}$ such that $y_{n} \lll e$ for all $n>N$. Thus, $x_{n} \preceq y_{n} \lll e$ for all $n>N$. Now, by Proposition 3, we have $x_{n} \lll e$ for all $n>N$.

Remark 2. Let $\left\{x_{n}\right\}$ and $\left\{y_{n}\right\}$ be two sequences in $E$ such that $x_{n} \preceq y_{n}$. Then $\left\{x_{n}\right\}$ is an e-sequence provided that $\left\{y_{n}\right\}$ is an e-sequence. Indeed, by the proof of Proposition 13, the claim holds.

Proposition 14. Let $0 \leq \lambda<1$ be a constant, $\left\{x_{n}\right\}$ and $\left\{y_{n}\right\}$ be sequences in $E^{+}$satisfying

$$
x_{n+1} \preceq \lambda x_{n}+y_{n} .
$$

Then $\left\{x_{n}\right\}$ is an e-sequence if $\left\{y_{n}\right\}$ is an e-sequence.

Proof. Assume that $\left\{y_{n}\right\}$ is an $e$-sequence. Then for any $e \ggg \theta_{E}$, there exists $N_{1} \in \mathbb{N}$ such that

$$
y_{n} \lll \frac{1-\lambda}{2} e
$$

for all $n>N_{1}$. Note that $\lambda^{n-N_{1}} x_{N_{1}+1} \rightarrow \theta_{E}(n \rightarrow \infty)$. Then by Proposition 12 , there exists $N_{2} \in \mathbb{N}$ such that

$$
\lambda^{n-N_{1}} x_{N_{1}+1} \lll \frac{1}{2} e
$$

for all $n>N_{2}$.

Put $N=\max \left\{N_{1}, N_{2}\right\}$, then (8) and (9) hold for all $n>N$. Using (7), we have

$$
\begin{aligned}
x_{n+1}-\lambda x_{n} & \preceq y_{n}, \\
\lambda x_{n}-\lambda^{2} x_{n-1} & \preceq \lambda y_{n-1}, \\
\lambda^{2} x_{n-1}-\lambda^{3} x_{n-2} & \preceq \lambda^{2} y_{n-2}, \\
\cdots \cdots \cdots \cdots \cdots \cdots & \ldots \cdots \cdots \\
\lambda^{n-N_{1}-1} x_{N_{1}+2}-\lambda^{n-N_{1}} x_{N_{1}+1} & \preceq \lambda^{n-N_{1}-1} y_{N_{1}+1} .
\end{aligned}
$$

Consider the above inequalities, for all $n>N$, it follows that

$$
\begin{aligned}
x_{n+1} & \preceq \lambda^{n-N_{1}} x_{N_{1}+1}+y_{n}+\lambda y_{n-1}+\lambda^{2} y_{n-2}+\cdots+\lambda^{n-N_{1}-1} y_{N_{1}+1} \\
& \lll \frac{1}{2} e+\left(1+\lambda+\lambda^{2}+\cdots+\lambda^{n-N_{1}-1}\right) \cdot \frac{1-\lambda}{2} e \\
& \lll \frac{1}{2} e+\frac{1}{1-\lambda} \cdot \frac{1-\lambda}{2} e \\
& =e .
\end{aligned}
$$

Finally, by Proposition 3, we claim that $\left\{x_{n}\right\}$ is an $e$-sequence.

Proposition 15. Let $\left(X, d^{E}\right)$ be an E-metric space and $\left\{x_{n}\right\}$ a sequence in $X$ satisfying

$$
d^{E}\left(x_{n}, x_{n+1}\right) \preceq \lambda d^{E}\left(x_{n-1}, x_{n}\right) \quad(n=1,2, \ldots),
$$

where $0 \leq \lambda<1$ is a constant. Then $\left\{x_{n}\right\}$ is an e-Cauchy sequence in $X$.

Proof. By (10), it follows that

$$
d^{E}\left(x_{n}, x_{n+1}\right) \preceq \lambda d^{E}\left(x_{n-1}, x_{n}\right) \preceq \lambda^{2} d^{E}\left(x_{n-2}, x_{n-1}\right) \preceq \cdots \preceq \lambda^{n} d^{E}\left(x_{0}, x_{1}\right) .
$$


For any $n, m \in \mathbb{N}$ and $m>n$, using (11), we have

$$
\begin{aligned}
d^{E}\left(x_{n}, x_{m}\right) & \preceq d^{E}\left(x_{n}, x_{n+1}\right)+d^{E}\left(x_{n+1}, x_{n+2}\right)+\cdots+d^{E}\left(x_{m-1}, x_{m}\right) \\
& \preceq\left(\lambda^{n}+\lambda^{n+1}+\cdots+\lambda^{m-1}\right) d^{E}\left(x_{0}, x_{1}\right) \\
& \preceq \frac{\lambda^{n}}{1-\lambda} d^{E}\left(x_{0}, x_{1}\right) \rightarrow \theta_{E}(n \rightarrow \infty) .
\end{aligned}
$$

Then by (12) and Proposition 13, we claim that $\left\{x_{n}\right\}$ is an $e$-Cauchy sequence in $X$.

Proposition 16. Let $\left(X, d^{E}\right)$ be an E-metric space wherein $(E,\|\cdot\|)$ is complete, $\left\{x_{n}\right\}$ and $\left\{y_{n}\right\}$ be e-Cauchy sequences in $X$. Then $E^{+}$is not a normal cone provided that $\left\{d^{E}\left(x_{n}, y_{n}\right)\right\}$ is not convergent in $E$.

Proof. Assume that $\left\{d^{E}\left(x_{n}, y_{n}\right)\right\}$ is not convergent in $E$. We argue by contradiction by supposing that $E^{+}$is a normal cone with the normal constant $M$. We start with $\varepsilon>0$ and take $e \in\left(E^{+}\right)^{\ominus}$ with $\|e\|<\frac{2 \varepsilon}{2 M+1}$. Since $\left\{x_{n}\right\}$ is an $e$-Cauchy sequence, then there exists $N_{1} \in \mathbb{N}$ such that

$$
d^{E}\left(x_{n}, x_{m}\right) \lll \frac{e}{4},
$$

for all $n, m>N_{1}$. Since $\left\{y_{n}\right\}$ is an $e$-Cauchy sequence, then there exists $N_{2} \in \mathbb{N}$ such that

$$
d^{E}\left(y_{n}, y_{m}\right) \lll \frac{e}{4},
$$

for all $n, m>N_{2}$. Let $N=\max \left\{N_{1}, N_{2}\right\}$, then (13) and (14) hold for all $n, m>N$. Accordingly, for all $n, m>N$, we deduce that

$$
\begin{aligned}
& d^{E}\left(x_{n}, y_{n}\right) \preceq d^{E}\left(x_{n}, x_{m}\right)+d^{E}\left(x_{m}, y_{m}\right)+d^{E}\left(y_{m}, y_{n}\right) \lll d^{E}\left(x_{m}, y_{m}\right)+\frac{e}{2}, \\
& d^{E}\left(x_{m}, y_{m}\right) \preceq d^{E}\left(x_{m}, x_{n}\right)+d^{E}\left(x_{n}, y_{n}\right)+d^{E}\left(y_{n}, y_{m}\right) \lll d^{E}\left(x_{n}, y_{n}\right)+\frac{e}{2} .
\end{aligned}
$$

Combining (15) and (16), we have

$$
\theta_{E} \lll d^{E}\left(x_{m}, y_{m}\right)+\frac{e}{2}-d^{E}\left(x_{n}, y_{n}\right) \lll d^{E}\left(x_{n}, y_{n}\right)+\frac{e}{2}+\frac{e}{2}-d^{E}\left(x_{n}, y_{n}\right)=e .
$$

By Proposition 1, (17) establishes that

$$
\theta_{E} \preceq d^{E}\left(x_{m}, y_{m}\right)+\frac{e}{2}-d^{E}\left(x_{n}, y_{n}\right) \preceq e .
$$

Since $E^{+}$is a normal cone, then it may be verified from (18) that

$$
\left\|d^{E}\left(x_{m}, y_{m}\right)+\frac{e}{2}-d^{E}\left(x_{n}, y_{n}\right)\right\| \leq M\|e\|
$$

Hence, by using (19), we obtain

$$
\left\|d^{E}\left(x_{m}, y_{m}\right)-d^{E}\left(x_{n}, y_{n}\right)\right\| \leq\left\|d^{E}\left(x_{m}, y_{m}\right)+\frac{e}{2}-d^{E}\left(x_{n}, y_{n}\right)\right\|+\left\|\frac{e}{2}\right\| \leq\left(M+\frac{1}{2}\right)\|e\|<\varepsilon,
$$

which means that $\left\{d^{E}\left(x_{n}, y_{n}\right)\right\}$ is a Cauchy sequence in $E$. Since $(E,\|\cdot\|)$ is complete, then $\left\{d^{E}\left(x_{n}, y_{n}\right)\right\}$ is convergent. This leads to a contradiction with the hypothesis.

Theorem 1. Let $\left(X, d^{E}\right)$ be an E-metric space and $\left\{x_{n}\right\}$ a sequence in $X$. Then the following are equivalent: (1) $\left(X, d^{E}\right)$ is e-complete; 
(2) there exists a unique point $p \in \bigcap_{n=1}^{\infty} S_{n}$ provided that $\left\{r_{n}\right\}$ is an e-sequence where $S_{n}=\{x \in X$ : $\left.d^{E}\left(x_{n}, x\right) \preceq r_{n}\right\}$ with $S_{1} \supseteq S_{2} \supseteq \cdots \supseteq S_{n} \supseteq \cdots$.

Proof. Let (1) hold, $\left\{r_{n}\right\}$ be an $e$-sequence and $S_{n}=\left\{x \in X: d^{E}\left(x_{n}, x\right) \preceq r_{n}\right\}$ with $S_{1} \supseteq S_{2} \supseteq$ $\cdots \supseteq S_{n} \supseteq \cdots$. In view of $x_{m} \in S_{m} \subseteq S_{n}(m \geq n)$, it follows that $d^{E}\left(x_{n}, x_{m}\right) \preceq r_{n}$. Since $\left\{r_{n}\right\}$ is an $e$-sequence, then by Proposition 3 or Remark 2, it is easy to see that $\left\{x_{n}\right\}$ is an $e$-Cauchy sequence in $X$. Since $\left(X, d^{E}\right)$ is $e$-complete, then there exists $p \in X$ such that $\left\{x_{n}\right\} e$-converges to $p$. In other words, for every $e \ggg \theta_{E}$ and any $k \in \mathbb{N}$, there exists $N_{1} \in \mathbb{N}$ such that for all $m>N_{1}$, one has $d^{E}\left(x_{m}, p\right) \lll \frac{e}{k}$. As a consequence of

$$
d^{E}\left(x_{n}, p\right) \preceq d^{E}\left(x_{n}, x_{m}\right)+d^{E}\left(x_{m}, p\right) \preceq r_{n}+d^{E}\left(x_{m}, p\right) \lll r_{n}+\frac{e}{k^{\prime}}
$$

we get from Proposition 3 that $r_{n}+\frac{e}{k}-d^{E}\left(x_{n}, p\right) \in\left(E^{+}\right)^{\ominus}$. Using Proposition 8 and letting $k \rightarrow \infty$ from this item, we have $r_{n}-d^{E}\left(x_{n}, p\right) \in\left(E^{+}\right)^{\ominus}$, further, by Definition 6, we have $r_{n}-d^{E}\left(x_{n}, p\right) \in E^{+}$, that is, $d^{E}\left(x_{n}, p\right) \preceq r_{n}$, i.e., $p \in S_{n}$. Thus, $p \in \bigcap_{n=1}^{\infty} S_{n}$.

On the other hand, if there exists $q \in \bigcap_{n=1}^{\infty} S_{n}$, then $d^{E}\left(x_{n}, q\right) \preceq r_{n}$. For each $e \ggg \theta_{E}$, since $\left\{r_{n}\right\}$ is an $e$-sequence and $\left\{x_{n}\right\} e$-converges to $p$, then there exists $N_{2} \in \mathbb{N}$ such that for all $n>N_{2}$, we get $r_{n} \lll \frac{e}{2}$ and $d^{E}\left(x_{n}, p\right) \lll \frac{e}{2}$. Consequently, for all $n>N_{2}$, we deduce that

$$
d^{E}(q, p) \preceq d^{E}\left(q, x_{n}\right)+d^{E}\left(x_{n}, p\right) \preceq r_{n}+d^{E}\left(x_{n}, p\right) \lll \frac{e}{2}+\frac{e}{2}=e,
$$

so by Propositions 3 and 7 , we obtain $d^{E}(q, p)=\theta_{E}$, i.e., $q=p$.

Conversely, assume that (2) holds and $\left\{x_{n}\right\}$ is an $e$-Cauchy sequence in $X$. Under this hypothesis, we may choose $e_{0} \in\left(E^{+}\right)^{\ominus}$ and $n_{1}<n_{2}<\cdots<n_{k}<\cdots$ satisfying

$$
d^{E}\left(x_{n}, x_{m}\right) \lll \frac{e_{0}}{2^{k+1}}
$$

for all $n, m \geq n_{k}$. We denote

$$
S\left(x_{n_{k}}, \frac{e_{0}}{2^{k}}\right)=\left\{x \in X: d^{E}\left(x_{n_{k}}, x\right) \preceq \frac{e_{0}}{2^{k}}\right\}, \quad k=1,2, \ldots,
$$

and take $y \in S\left(x_{n_{k+1}}, \frac{e_{0}}{2^{k+1}}\right)$. As

$$
d^{E}\left(x_{n_{k}}, y\right) \preceq d^{E}\left(x_{n_{k}}, x_{n_{k+1}}\right)+d^{E}\left(x_{n_{k+1}}, y\right) \lll \frac{e_{0}}{2^{k+1}}+\frac{e_{0}}{2^{k+1}}=\frac{e_{0}}{2^{k}}
$$

by Propositions 1 and 3, we speculate $y \in S\left(x_{n_{k}}, \frac{e_{0}}{2^{k}}\right)$, thus, $S\left(x_{n_{k+1}}, \frac{e_{0}}{2^{k+1}}\right) \subseteq S\left(x_{n_{k}}, \frac{e_{0}}{2^{k}}\right)$. By virtue of (2), there is a unique point $p \in \bigcap_{k=1}^{\infty} S\left(x_{n_{k^{\prime}}}, \frac{e_{0}}{2^{k}}\right)$. Hence, $d^{E}\left(x_{n_{k}}, p\right) \preceq \frac{e_{0}}{2^{k}}$. Since $\left\{x_{n}\right\}$ is an $e$-Cauchy sequence, then for each $e \ggg \theta_{E}$, there exists $N_{3} \in \mathbb{N}$ such that for all $n, m>N_{3}$, one has $d^{E}\left(x_{n}, x_{m}\right) \lll \frac{e}{2}$. Note that $d^{E}\left(x_{n_{k}}, p\right) \preceq \frac{e_{0}}{2^{k}} \rightarrow \theta_{E}(k \rightarrow \infty)$, then for each $e \ggg \theta_{E}$, by Proposition 13, there exists $k_{0}$ such that $n_{k_{0}}>N_{3}$ and $d^{E}\left(x_{n_{k_{0}}}, p\right) \lll \frac{e}{2}$. Consequently, for all $n>N_{3}$, we have

$$
d^{E}\left(x_{n}, p\right) \preceq d^{E}\left(x_{n}, x_{n_{k_{0}}}\right)+d^{E}\left(x_{n_{k_{0}}}, p\right) \lll \frac{e}{2}+\frac{e}{2}=e,
$$

which follows from Proposition 3 that $\left\{x_{n}\right\}$ e-converges to $p$. Therefore, $\left(X, d^{E}\right)$ is $e$-complete. 
Remark 3. Theorem 1 gives us a sufficient and necessary condition on e-completeness in the setting of E-metric spaces. It is regarded as the theorem of nested closed-ball in E-metric spaces. Clearly, it expands the theorem of nested interval from metric spaces to E-metric spaces.

\section{Some Applications to Fixed Point Theory}

In this section, we use the aforementioned topological properties to deal with a class of fixed point problems. As compared to the previous methods, our results are more general and the proofs are more straightforward.

Similar to Definition 2.24 of [11], we introduce the following notion.

Definition 9. Let $\left(X, d^{E}\right)$ be an E-metric space, $\left\{y_{n}\right\}$ a sequence in $X$ and $T$ a self-map on $X$. Let $x_{0} \in X$ and $x_{n+1}=T x_{n}$ be a Picard's iteration in X. The iteration procedure $x_{n+1}=T x_{n}$ is said to be T-stable with respect to $T$ if $\left\{x_{n}\right\}$ e-converges to a fixed point $q$ of $T$, and $\left\{d^{E}\left(y_{n+1}, T y_{n}\right)\right\}$ is an e-sequence, then $\left\{y_{n}\right\}$ e-converges to $q$.

Subsequently, motivated by Theorem 1 of [16], we introduce the concept of Hardy-Rogers type mapping in the framework of $E$-metric spaces.

Definition 10. Let $\left(X, d^{E}\right)$ be an E-metric space and $T: X \rightarrow X$ be a mapping satisfying

$$
\begin{aligned}
d^{E}(T x, T y) \preceq & \lambda_{1} d^{E}(x, y)+\lambda_{2} d^{E}(x, T x)+\lambda_{3} d^{E}(y, T y) \\
& +\lambda_{4} d^{E}(x, T y)+\lambda_{5} d^{E}(y, T x)
\end{aligned}
$$

for all $x, y \in X$, where $\lambda_{i} \geq 0(i=1,2,3,4,5)$ are constants such that $0 \leq \sum_{i=1}^{5} \lambda_{i}<1$. Then $T$ is called a Hardy-Rogers type mapping on $X$.

Finally, taking advantage of the above topological properties, we give some applications to fixed point theory with respect to Hardy-Rogers type mappings on $E$-metric spaces.

Theorem 2. Let $\left(X, d^{E}\right)$ be an e-complete E-metric space and $T: X \rightarrow X$ a Hardy-Rogers type mapping on $X$. Then

(1) Thas a unique fixed point in $X$, and for each $x \in X$, the iterative sequence $\left\{T^{n} x\right\}_{n \geq 0}$ e-converges to the fixed point;

(2) the Picard's iteration is T-stable;

(3) $\left\{d\left(y_{n}, T y_{n}\right)\right\}$ is an e-sequence if and only if $\left\{d\left(y_{n+1}, T y_{n}\right)\right\}$ is an e-sequence.

Proof. (1) We choose $x_{0} \in X$ and construct the Picard's iterative sequence $\left\{x_{n}\right\}$ by $x_{n+1}=T x_{n}=$ $T^{n+1} x_{0}$. Taking advantage of (20), on the one hand, we have

$$
\begin{aligned}
d^{E}\left(x_{n}, x_{n+1}\right)= & d^{E}\left(T x_{n-1}, T x_{n}\right) \\
\preceq & \lambda_{1} d^{E}\left(x_{n-1}, x_{n}\right)+\lambda_{2} d^{E}\left(x_{n-1}, T x_{n-1}\right)+\lambda_{3} d^{E}\left(x_{n}, T x_{n}\right) \\
& +\lambda_{4} d^{E}\left(x_{n-1}, T x_{n}\right)+\lambda_{5} d^{E}\left(x_{n}, T x_{n-1}\right) \\
= & \left(\lambda_{1}+\lambda_{2}\right) d^{E}\left(x_{n-1}, x_{n}\right)+\lambda_{3} d^{E}\left(x_{n}, x_{n+1}\right)+\lambda_{4} d^{E}\left(x_{n-1}, x_{n+1}\right) \\
\preceq & \left(\lambda_{1}+\lambda_{2}+\lambda_{4}\right) d^{E}\left(x_{n-1}, x_{n}\right)+\left(\lambda_{3}+\lambda_{4}\right) d^{E}\left(x_{n}, x_{n+1}\right),
\end{aligned}
$$

on the other hand, we have

$$
\begin{aligned}
d^{E}\left(x_{n}, x_{n+1}\right) & =d^{E}\left(T x_{n}, T x_{n-1}\right) \\
& \preceq \lambda_{1} d^{E}\left(x_{n}, x_{n-1}\right)+\lambda_{2} d^{E}\left(x_{n}, T x_{n}\right)+\lambda_{3} d^{E}\left(x_{n-1}, T x_{n-1}\right)
\end{aligned}
$$




$$
\begin{aligned}
& +\lambda_{4} d^{E}\left(x_{n}, T x_{n-1}\right)+\lambda_{5} d^{E}\left(x_{n-1}, T x_{n}\right) \\
= & \left(\lambda_{1}+\lambda_{3}\right) d^{E}\left(x_{n-1}, x_{n}\right)+\lambda_{2} d^{E}\left(x_{n}, x_{n+1}\right)+\lambda_{5} d^{E}\left(x_{n-1}, x_{n+1}\right) \\
\preceq & \left(\lambda_{1}+\lambda_{3}+\lambda_{5}\right) d^{E}\left(x_{n-1}, x_{n}\right)+\left(\lambda_{2}+\lambda_{5}\right) d^{E}\left(x_{n}, x_{n+1}\right) .
\end{aligned}
$$

Adding up (21) and (22) yields

$$
\begin{aligned}
2 d^{E}\left(x_{n}, x_{n+1}\right) & \preceq\left(2 \lambda_{1}+\lambda_{2}+\lambda_{3}+\lambda_{4}+\lambda_{5}\right) d^{E}\left(x_{n-1}, x_{n}\right) \\
& +\left(\lambda_{2}+\lambda_{3}+\lambda_{4}+\lambda_{5}\right) d^{E}\left(x_{n}, x_{n+1}\right),
\end{aligned}
$$

from which follows

$$
d^{E}\left(x_{n}, x_{n+1}\right) \preceq \frac{2 \lambda_{1}+\lambda_{2}+\lambda_{3}+\lambda_{4}+\lambda_{5}}{2-\lambda_{2}-\lambda_{3}-\lambda_{4}-\lambda_{5}} d^{E}\left(x_{n-1}, x_{n}\right) .
$$

We make $\lambda=\frac{2 \lambda_{1}+\lambda_{2}+\lambda_{3}+\lambda_{4}+\lambda_{5}}{2-\lambda_{2}-\lambda_{3}-\lambda_{4}-\lambda_{5}}$, then $0 \leq \lambda<1$ by right of $0 \leq \sum_{i=1}^{5} \lambda_{i}<1$. Equation (23) and Proposition 15 ensure us that $\left\{x_{n}\right\}$ is an $e$-Cauchy sequence in $X$.

Since $\left(X, d^{E}\right)$ is $e$-complete, then there exists $q \in X$ such that $\left\{x_{n}\right\} e$-converges to $q$. In the following, we show that $q$ is a fixed point of $T$.

Indeed, using (20), we speculate that

$$
\begin{aligned}
d^{E}(T q, q) \preceq & d^{E}\left(T q, T x_{n-1}\right)+d^{E}\left(x_{n}, q\right) \\
\preceq & \lambda_{1} d^{E}\left(q, x_{n-1}\right)+\lambda_{2} d^{E}(q, T q)+\lambda_{3} d^{E}\left(x_{n-1}, T x_{n-1}\right) \\
& +\lambda_{4} d^{E}\left(q, T x_{n-1}\right)+\lambda_{5} d^{E}\left(x_{n-1}, T q\right)+d^{E}\left(x_{n}, q\right) \\
\preceq & \left(\lambda_{1}+\lambda_{3}+\lambda_{5}\right) d^{E}\left(x_{n-1}, q\right)+\left(\lambda_{2}+\lambda_{5}\right) d(T q, q)+\left(1+\lambda_{3}+\lambda_{4}\right) d^{E}\left(x_{n}, q\right),
\end{aligned}
$$

which means that

$$
d^{E}(T q, q) \preceq \frac{\lambda_{1}+\lambda_{3}+\lambda_{5}}{1-\lambda_{2}-\lambda_{5}} d^{E}\left(x_{n-1}, q\right)+\frac{1+\lambda_{3}+\lambda_{4}}{1-\lambda_{2}-\lambda_{5}} d^{E}\left(x_{n}, q\right) \triangleq z_{n} .
$$

Since $\left\{x_{n}\right\}$ e-converges to $q$, then $\left\{d^{E}\left(x_{n}, q\right)\right\}$ is an $e$-sequence. Thus, by Proposition $11,\left\{z_{n}\right\}$ is also an $e$-sequence. Therefore, by (24) and Proposition 3, for any $e \ggg \theta_{E}$, there exists $N \in \mathbb{N}$ such that for all $n>N$, ones have

$$
d^{E}(T q, q) \lll e .
$$

Accordingly, by (25) and Proposition 7, it is obvious that $d^{E}(T q, q)=\theta_{E}$, i.e., $q$ is a fixed point of $T$.

Now we prove that the fixed point of $T$ is unique. To this end, assume that there exists another fixed point $p$ of $T$. Then by utilizing (20), it follows that

$$
\begin{aligned}
d^{E}(q, p)= & d^{E}(T q, T p) \\
\preceq & \lambda_{1} d^{E}(q, p)+\lambda_{2} d^{E}(q, T q)+\lambda_{3} d^{E}(p, T p) \\
& +\lambda_{4} d^{E}(q, T p)+\lambda_{5} d^{E}(p, T q) \\
= & \left(\lambda_{1}+\lambda_{4}+\lambda_{5}\right) d^{E}(q, p) .
\end{aligned}
$$

As $0 \leq \lambda_{1}+\lambda_{4}+\lambda_{5} \leq \sum_{i=1}^{5} \lambda_{i}<1$, then by Lemma 1 , we get $d^{E}(q, p)=\theta_{E}$. Hence, $q=p$.

(2) Assume that $\left\{y_{n}\right\}$ is a sequence in $X$ such that $\left\{d\left(y_{n+1}, T y_{n}\right)\right\}$ is an $e$-sequence. Using (20), firstly we have

$$
d^{E}\left(T y_{n}, q\right)=d^{E}\left(T y_{n}, T q\right)
$$




$$
\begin{aligned}
\preceq & \lambda_{1} d^{E}\left(y_{n}, q\right)+\lambda_{2} d^{E}\left(y_{n}, T y_{n}\right)+\lambda_{3} d^{E}(q, T q) \\
& +\lambda_{4} d^{E}\left(y_{n}, T q\right)+\lambda_{5} d^{E}\left(q, T y_{n}\right) \\
= & \lambda_{1} d^{E}\left(y_{n}, q\right)+\lambda_{2} d^{E}\left(y_{n}, T y_{n}\right)+\lambda_{4} d^{E}\left(y_{n}, q\right)+\lambda_{5} d^{E}\left(q, T y_{n}\right) \\
\preceq & \left(\lambda_{1}+\lambda_{4}\right) d^{E}\left(y_{n}, q\right)+\lambda_{2}\left[d^{E}\left(y_{n}, q\right)+d^{E}\left(q, T y_{n}\right)\right]+\lambda_{5} d^{E}\left(q, T y_{n}\right) \\
= & \left(\lambda_{1}+\lambda_{2}+\lambda_{4}\right) d^{E}\left(y_{n}, q\right)+\left(\lambda_{2}+\lambda_{5}\right) d^{E}\left(q, T y_{n}\right) .
\end{aligned}
$$

Secondly, we obtain that

$$
\begin{aligned}
d^{E}\left(T y_{n}, q\right)= & d^{E}\left(q, T y_{n}\right)=d^{E}\left(T q, T y_{n}\right) \\
\preceq & \lambda_{1} d^{E}\left(q, y_{n}\right)+\lambda_{2} d^{E}(q, T q)+\lambda_{3} d^{E}\left(y_{n}, T y_{n}\right) \\
& +\lambda_{4} d^{E}\left(q, T y_{n}\right)+\lambda_{5} d^{E}\left(y_{n}, T q\right) \\
= & \lambda_{1} d^{E}\left(y_{n}, q\right)+\lambda_{3} d^{E}\left(y_{n}, T y_{n}\right)+\lambda_{4} d^{E}\left(q, T y_{n}\right)+\lambda_{5} d^{E}\left(y_{n}, q\right) \\
\preceq & \left(\lambda_{1}+\lambda_{5}\right) d^{E}\left(y_{n}, q\right)+\lambda_{3}\left[d^{E}\left(y_{n}, q\right)+d^{E}\left(q, T y_{n}\right)\right]+\lambda_{4} d^{E}\left(q, T y_{n}\right) \\
= & \left(\lambda_{1}+\lambda_{3}+\lambda_{5}\right) d^{E}\left(y_{n}, q\right)+\left(\lambda_{3}+\lambda_{4}\right) d^{E}\left(q, T y_{n}\right) .
\end{aligned}
$$

We add up (26) and (27), which yields

$$
2 d^{E}\left(T y_{n}, q\right) \preceq\left(2 \lambda_{1}+\lambda_{2}+\lambda_{3}+\lambda_{4}+\lambda_{5}\right) d^{E}\left(y_{n}, q\right)+\left(\lambda_{2}+\lambda_{3}+\lambda_{4}+\lambda_{5}\right) d^{E}\left(q, T y_{n}\right),
$$

which means that

$$
d^{E}\left(T y_{n}, q\right) \preceq \frac{2 \lambda_{1}+\lambda_{2}+\lambda_{3}+\lambda_{4}+\lambda_{5}}{2-\lambda_{2}-\lambda_{3}-\lambda_{4}-\lambda_{5}} d^{E}\left(y_{n}, q\right) .
$$

As mentioned above, on account of $\lambda=\frac{2 \lambda_{1}+\lambda_{2}+\lambda_{3}+\lambda_{4}+\lambda_{5}}{2-\lambda_{2}-\lambda_{3}-\lambda_{4}-\lambda_{5}}$, then $0 \leq \lambda<1$ and

$$
d^{E}\left(T y_{n}, q\right) \preceq \lambda d^{E}\left(y_{n}, q\right) .
$$

Now, setting $a_{n}=d^{E}\left(y_{n}, q\right)$ and $c_{n}=d^{E}\left(y_{n+1}, T y_{n}\right)$, we establish

$$
a_{n+1}=d^{E}\left(y_{n+1}, q\right) \preceq d^{E}\left(y_{n+1}, T y_{n}\right)+d^{E}\left(T y_{n}, q\right) \preceq \lambda a_{n}+c_{n} .
$$

Since $\left\{c_{n}\right\}$ is an $e$-sequence, using Proposition 14 , we deduce that $\left\{a_{n}\right\}$ is an $e$-sequence. Thus, $\left\{y_{n}\right\}$ $e$-converges to $q$ as $n \rightarrow \infty$. This implies that the Picard's iteration is $T$-stable.

(3) Suppose that $\left\{y_{n}\right\}$ is a sequence in X. Put $b_{n}=d^{E}\left(y_{n}, T y_{n}\right)$. If $\left\{c_{n}\right\}$ is an $e$-sequence, then for one thing, we have

$$
\begin{aligned}
b_{n}= & d^{E}\left(y_{n}, T y_{n}\right) \preceq d^{E}\left(y_{n}, T y_{n-1}\right)+d^{E}\left(T y_{n}, T y_{n-1}\right) \\
\preceq & d^{E}\left(y_{n}, T y_{n-1}\right)+\lambda_{1} d^{E}\left(y_{n}, y_{n-1}\right)+\lambda_{2} d^{E}\left(y_{n}, T y_{n}\right)+\lambda_{3} d^{E}\left(y_{n-1}, T y_{n-1}\right) \\
& +\lambda_{4} d^{E}\left(y_{n}, T y_{n-1}\right)+\lambda_{5} d^{E}\left(y_{n-1}, T y_{n}\right) \\
& \preceq d^{E}\left(y_{n}, T y_{n-1}\right)+\lambda_{1}\left[d^{E}\left(y_{n}, T y_{n-1}\right)+d^{E}\left(T y_{n-1}, y_{n-1}\right)\right] \\
& +\lambda_{2} d^{E}\left(y_{n}, T y_{n}\right)+\lambda_{3} d^{E}\left(y_{n-1}, T y_{n-1}\right)+\lambda_{4} d^{E}\left(y_{n}, T y_{n-1}\right) \\
& +\lambda_{5}\left[d^{E}\left(y_{n-1}, T y_{n-1}\right)+d^{E}\left(T y_{n-1}, y_{n}\right)+d^{E}\left(y_{n}, T y_{n}\right)\right] \\
& =\left(\lambda_{2}+\lambda_{5}\right) b_{n}+\left(\lambda_{1}+\lambda_{3}+\lambda_{5}\right) b_{n-1}+\left(1+\lambda_{1}+\lambda_{4}+\lambda_{5}\right) c_{n-1},
\end{aligned}
$$

which implies that

$$
\left(1-\lambda_{2}-\lambda_{5}\right) b_{n} \preceq\left(\lambda_{1}+\lambda_{3}+\lambda_{5}\right) b_{n-1}+\left(1+\lambda_{1}+\lambda_{4}+\lambda_{5}\right) c_{n-1} .
$$


For another thing, we have

$$
\begin{aligned}
b_{n}= & d^{E}\left(y_{n}, T y_{n}\right) \preceq d^{E}\left(y_{n}, T y_{n-1}\right)+d^{E}\left(T y_{n-1}, T y_{n}\right) \\
\preceq & d^{E}\left(y_{n}, T y_{n-1}\right)+\lambda_{1} d^{E}\left(y_{n-1}, y_{n}\right)+\lambda_{2} d^{E}\left(y_{n-1}, T y_{n-1}\right)+\lambda_{3} d^{E}\left(y_{n}, T y_{n}\right) \\
& +\lambda_{4} d^{E}\left(y_{n-1}, T y_{n}\right)+\lambda_{5} d^{E}\left(y_{n}, T y_{n-1}\right) \\
& d^{E}\left(y_{n}, T y_{n-1}\right)+\lambda_{1}\left[\left(d^{E}\left(y_{n-1}, T y_{n-1}\right)+d^{E}\left(T y_{n-1}, y_{n}\right)\right]\right. \\
& +\lambda_{2} d^{E}\left(y_{n-1}, T y_{n-1}\right)+\lambda_{3} d^{E}\left(y_{n}, T y_{n}\right)+\lambda_{5} d^{E}\left(y_{n}, T y_{n-1}\right) \\
& +\lambda_{4}\left[d^{E}\left(y_{n-1}, T y_{n-1}\right)+d^{E}\left(T y_{n-1}, y_{n}\right)+d^{E}\left(y_{n}, T y_{n}\right)\right] \\
& =\left(\lambda_{3}+\lambda_{4}\right) b_{n}+\left(\lambda_{1}+\lambda_{2}+\lambda_{4}\right) b_{n-1}+\left(1+\lambda_{1}+\lambda_{4}+\lambda_{5}\right) c_{n-1},
\end{aligned}
$$

which establishes that

$$
\left(1-\lambda_{3}-\lambda_{4}\right) b_{n} \preceq\left(\lambda_{1}+\lambda_{2}+\lambda_{4}\right) b_{n-1}+\left(1+\lambda_{1}+\lambda_{4}+\lambda_{5}\right) c_{n-1} .
$$

Sum up both (28) and (29) and it follows that

$$
b_{n} \preceq \frac{2 \lambda_{1}+\lambda_{2}+\lambda_{3}+\lambda_{4}+\lambda_{5}}{2-\lambda_{2}-\lambda_{3}-\lambda_{4}-\lambda_{5}} b_{n-1}+\frac{2\left(1+\lambda_{1}+\lambda_{4}+\lambda_{5}\right)}{2-\lambda_{2}-\lambda_{3}-\lambda_{4}-\lambda_{5}} c_{n-1} .
$$

Noticing that $0 \leq \lambda=\frac{2 \lambda_{1}+\lambda_{2}+\lambda_{3}+\lambda_{4}+\lambda_{5}}{2-\lambda_{2}-\lambda_{3}-\lambda_{4}-\lambda_{5}}<1$, we deduce from Proposition 14 that $\left\{b_{n}\right\}$ is an $e$-sequence.

Conversely, if $\left\{b_{n}\right\}$ is an $e$-sequence, then

$$
\begin{aligned}
c_{n}= & d^{E}\left(y_{n+1}, T y_{n}\right) \preceq d^{E}\left(y_{n+1}, T y_{n+1}\right)+d^{E}\left(T y_{n+1}, T y_{n}\right) \\
\preceq & d^{E}\left(y_{n+1}, T y_{n+1}\right)+\lambda_{1} d^{E}\left(y_{n+1}, y_{n}\right)+\lambda_{2} d^{E}\left(y_{n+1}, T y_{n+1}\right) \\
& +\lambda_{3} d^{E}\left(y_{n}, T y_{n}\right)+\lambda_{4} d^{E}\left(y_{n+1}, T y_{n}\right)+\lambda_{5} d^{E}\left(y_{n}, T y_{n+1}\right) \\
\preceq & d^{E}\left(y_{n+1}, T y_{n+1}\right)+\lambda_{1}\left[d^{E}\left(y_{n+1}, T y_{n}\right)+d^{E}\left(y_{n}, T y_{n}\right)\right] \\
& +\lambda_{2} d^{E}\left(y_{n+1}, T y_{n+1}\right)+\lambda_{3} d^{E}\left(y_{n}, T y_{n}\right)+\lambda_{4} d^{E}\left(y_{n+1}, T y_{n}\right) \\
& +\lambda_{5}\left[d^{E}\left(y_{n}, T y_{n}\right)+d^{E}\left(T y_{n}, y_{n+1}\right)+d^{E}\left(y_{n+1}, T y_{n+1}\right)\right] \\
& =\left(1+\lambda_{2}+\lambda_{5}\right) b_{n+1}+\left(\lambda_{1}+\lambda_{3}+\lambda_{5}\right) b_{n}+\left(\lambda_{1}+\lambda_{4}+\lambda_{5}\right) c_{n} .
\end{aligned}
$$

It is obvious that

$$
c_{n} \preceq \frac{1+\lambda_{2}+\lambda_{5}}{1-\lambda_{1}-\lambda_{4}-\lambda_{5}} b_{n+1}+\frac{\lambda_{1}+\lambda_{3}+\lambda_{5}}{1-\lambda_{1}-\lambda_{4}-\lambda_{5}} b_{n} \triangleq d_{n} .
$$

Since $\left\{b_{n}\right\}$ is an $e$-sequence, then by Proposition $11,\left\{d_{n}\right\}$ is an $e$-sequence. Thus, by Remark 2 , it is not hard to verify that $\left\{c_{n}\right\}$ is an $e$-sequence.

Remark 4. Item (1) of Theorem 2 greatly generalizes the main theorems of [10]. As a matter of fact, if we take $\lambda_{1}=\lambda \in[0,1), \lambda_{2}=\lambda_{3}=\lambda_{4}=\lambda_{5}=0$ in (20), then (1) of Theorem 2 becomes Theorem 1 of [10]; if we take $\lambda_{1}=\lambda_{4}=\lambda_{5}=0$ and $\lambda_{2}=\lambda_{3}=\lambda \in\left[0, \frac{1}{2}\right)$ in (20), then (1) of Theorem 2 becomes Theorem 2 of [10]; if we take $\lambda_{1}=\lambda_{2}=\lambda_{3}=0$ and $\lambda_{4}=\lambda_{5}=\lambda \in\left[0, \frac{1}{2}\right)$ in (20), then (1) of Theorem 2 becomes Theorem 3 of [10].

Funding: The research was partly supported by the Talent Initial Funding for Scientific Research of Chongqing Three Gorges University (20190020).

Acknowledgments: The author thanks the editor and the referees for their valuable comments and suggestions which improved greatly the quality of this paper.

Conflicts of Interest: The author declares no conflict of interest. 


\section{References}

1. Huang, L.G.; Zhang, X. Cone metric spaces and fixed point theorems of contractive mappings. J. Math. Anal. Appl. 2007, 332, 1468-1476. [CrossRef]

2. Aleksić, S.; Kadelburg, Z.; Mitrović, Z.D.; Radenović, S. A new survey: Cone metric spaces. J. Int. Math. Virt. Inst. 2019, 9, 93-121.

3. Du, W.-S. A note on cone metric fixed point theory and its equivalence. Nonlinear Anal. 2010, 72, $2259-2261$. [CrossRef]

4. Huang, H.P.; Kadelburg, Z.; Radenović, S. A note on some recent results about multivalued mappings in TVS-cone metric spaces. J. Adv. Math. Stud. 2016, 9, 330-337.

5. Janković, S.; Kadelburg, Z.; Radenović, S. On cone metric spaces: A survey. Nonlinear Anal. 2011, 74, 2591-2601. [CrossRef]

6. Kadelburg, Z.; Radenović, S. A note on various types of cones and fixed point results in cone metric spaces. Asian J. Math. Appl. 2013, 2013, ama0104.

7. Rezapour, S.; Hamlbarani, R. Some notes on the paper "Cone metric spaces and fixed point theorems of contractive mappings". J. Math. Anal. Appl. 2008, 345, 719-724. [CrossRef]

8. Rawashdeh, A.A.; Shatanawi, W.; Khandaqji, M. Normed ordered and E-metric spaces. Int. J. Math. Math. Sci. 2012, 2012, 272137. [CrossRef]

9. Basile, A.; Graziano, M.G.; Papadaki, M.; Polyrakis, I.A. Cones with semi-interior points and equilibrium. J. Math. Econ. 2017, 71, 36-48. [CrossRef]

10. Mehmood, N.; Rawashdeh, A.A.; Radenović, S. New fixed point results for E-metric spaces. Positivity 2019, 23, 1101-1111. [CrossRef]

11. Huang, H.P.; Deng, G.T.; Radenović, S. Some topological properties and fixed point results in cone metric spaces over Banach algebras. Positivity 2019, 23, 21-34. [CrossRef]

12. Huang, H.P.; Xu, S.Y. Some new topological properties in cone metric spaces. J. Math. PRC 2015, 35, 513-518.

13. Oner, T. Some topological properties of fuzzy cone metric spaces. J. Nonlinear Sci. Appl. 2016, 9, $799-805$. [CrossRef]

14. Rezapour, S.; Derafshpour, M.; Hamlbarani, R. A review on topological properties of cone metric spaces. J. Bone Jt. Surg. 2008, s2-s9, 615-632.

15. Đordević, M.; Đorić, D.; Kadelburg, Z.; Radenović, S.; Spasić, D. Fixed point results under c-distance in tvs-cone metric spaces. Fixed Point Theory Appl. 2011, 2011, 29. [CrossRef]

16. Hardy, G.E.; Rogers, T.D. A generalization of a fixed point theorem of Reich. Canad. Math. Bull. 1973, 16, 201-206. [CrossRef]

(C) 2019 by the authors. Licensee MDPI, Basel, Switzerland. This article is an open access article distributed under the terms and conditions of the Creative Commons Attribution (CC BY) license (http:/ / creativecommons.org/licenses/by/4.0/). 